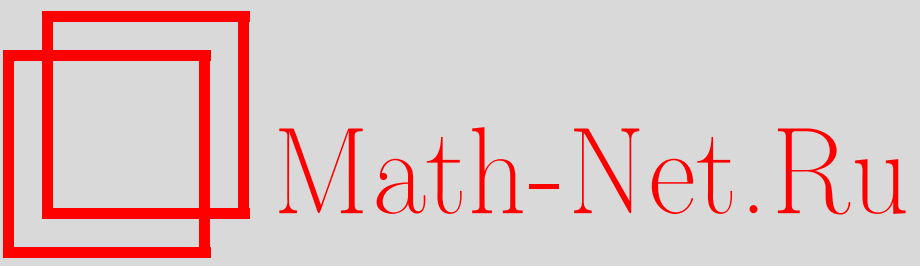

И. Ю. Типунин, Изоморфизм двумерных конформных теорий поля с аффинной $s l(2)$ и $N=2$ суперконформной симметриями, ТМФ, 1998, том 115, номер 1,29 45

DOI: https://doi.org/10.4213/tmf855

Использование Общероссийского математического портала Math-Net.Ru подразумевает, что вы прочитали и согласны с пользовательским соглашением

http://www.mathnet.ru/rus/agreement

Параметры загрузки:

IP : 3.85 .73 .92

26 апреля 2023 г., 17:27:36 
ТЕОРЕТИЧЕСКАЯ

И МАТЕМАТИЧЕСКАЯ

ФИЗИКА

Том 115, № 1

апрель, 1998

(C) 1998 г.

\section{ИЗОМОРФИЗМ ДВУМЕРНЫХ КОНФОРМНЫХ ТЕОРИЙ ПОЛЯ С АФФИННОЙ $s l(2)$ И $N=2$ СУПЕРКОНФОРМНОЙ СИММЕТРИЯМИ}

Обсуждается взаимно однозначное соответствие между двумерными конформными теориями поля с афффинной $s l(2)$ и $N=2$ суперконформной симметриями. Получены формулы, связывающие состояния с нулевой нормой двух теорий. Получены формулы, выражающие статистическую сумму теории с аффинной $s l(2)$-алгеброй симметрии через статистическую сумму теории с $N=2$ суперконформной алгеброй симметрии и наоборот.

\section{1. ВВЕДЕНИЕ}

В этой статье проводится анализ соответствия между двумерными конформными теориями поля, обладаюшими аффинной $s l(2)$ и $N=2$ суперконформной алгебрами симметрии. Обе эти теории встречаются во многих физических моделях. Для теорий с аффинной алгеброй симметрии $\widehat{s l}(2)$ достаточно указать на модели Весса-Зумино-Виттена [1] и подход Полякова к квантованию двумерной материи, одетой двумерной гравитацией $[2,3]$. Теории с $N=2$ суперконформной алгеброй симметрии также часто встречаются в физике. Среди теорий с $N=2$ симметрией отметим прежде всего теорию $N=2$ струн [4] и модели Ландау-Гинзбурга [5], а также модели двумерной гравитации $[6,7]$. В связи с этим особенно замечательно, что, как мы увидим ниже, модели, обладаюшие $s l(2)$ и $N=2$ симметриями, находятся во взаимно однозначном соответствии, и результаты, полученные в одной из них, переносятся на другую.

В дальнейшем будут активно использоваться различные понятия двумерной конформной теории поля, поэтому дадим краткое описание ключевых положений этой теории. Как известно [8], полная двумерная конформная теория (по модулю голоморфной аномалии, которая здесь обсуж даться не будет) распадается в прямую сумму голоморфной и антиголоморфной частей, в частности, алгебра наблюдаемых имеет вид $\mathcal{A} \oplus \overline{\mathcal{A}}$. В этой работе не рассматриваются вопросы комбинирования голоморфного и антиголоморфного секторов для получения полной модулярно инвариантной теории, а все рассмотрения ограничиваются голоморфным сектором. Среди полей теории должно при-

\footnotetext{
* Физический институт им. П. Н. Лебедева Российской академии наук, Москва, Россия
} 
сутствовать поле, называемое тензором энергии-импульса, которое имеет с самим собой фиксированное [8] операторное разложение такое, что моды тензора энергии-импульса образуют алгебру Вирасоро. В случае так называемых минимальных моделей [8] любое другое поле является либо примарным полем, либо вирасоровским потомком примарного поля. Алгеброй симметрии такой теории является алгебра Вирасоро. Пространство состояний теории представляет собой прямую сумму неприводимых представлений алгебры Вирасоро, которые играют роль секторов суперотбора теории, поскольку линейные комбинации состояний, принадлежащих разным неприводимым представлениям алгебры Вирасоро, не имеют смысла. По каждому примарному полю $\Phi(z)$ можно построить примарное состояние $\Phi(0)|0\rangle$, где $|0\rangle$ обозначает проективно-инвариантный вакуум [9] теории. Каждый сектор суперотбора порождается из примарного состояния действием операторов из алгебры Вирасоро, причем примарные состояния являются векторами старшего веса по отношению к алгебре Вирасоро. Конечно, сушествует много минимальных конформных теорий поля. Конкретная модель определяется выбором центрального заряда алгебры Вирасоро и набором примарных полей.

При изучении конкретной модели обычно интересуются ее коррелящионными функциями и статистической суммой. Корреляционные функции в двумерной конформной теории поля - это усредненные по вакууму $R$-произведения (аналог $T$-произведений) полей. Уравнения на корреляционные функции примарных полей получаются приравниванием к нулю состояний с нулевой нормой. Начиная с работ $[10,11]$ известно, когда и сколько существует в теории состояний с нулевой нормой и в случае, когда их сушествует "максимально возможное" число. Уравнения, полученные приравниванием к нулю этих состояний, определяют с точностью до констант все коррелящионные функции примарных полей.

Статистическая сумма является следом оператора $\exp \left\{2 \pi i \tau L_{0}+2 \pi i \bar{\tau} \bar{L}_{0}\right\}$, вычисленного по пространству состояний теории, где $L_{0}$ и $\bar{L}_{0}$ - голоморфная и антиголоморфная части гамильтониана модели (нулевые моды соответственно голоморфной и антиголоморфной частей тензора энергии-импульса). Полная статистическая сумма теории находится из условий модулярной инвариантности как билинейная комбинация следов операторов $e^{2 \pi i \tau L_{0}}$ и $e^{2 \pi i \bar{\tau} \bar{L}_{0}}$, вычисленных по каждому из секторов суперотбора. Каждый сектор суперотбора - это неприводимое представление алгебры Вирасоро, а след оператора $e^{2 \pi i \tau L_{0}}$, вычисленный по какому-нибудь представлению, называется характером этого представления.

В общем случае алгеброй симметрии является какая-нибудь алгебра, включающая в себя алгебру Вирасоро (точнее, универсальная обертывающая которой включает в себя универсальную обертывающую алгебры Вирасоро), например какая-нибудь аффинная алгебра Ли, $W$-алгебра, какое-нибудь суперсимметричное расширение алгебры Вирасоро и т.п. В этом случае пространство состояний будет прямой суммой неприводимых представлений выбранной алгебры симметрии. Аналогично случаю алгебры Вирасоро теория фиксируется заданием набора полей, примарных по отношению к рассматриваемой алгебре симметрии, и центральных зарядов алгебры. Уравнения на корреляционные функции также получаются приравниванием к нулю состояний с ну- 
левой нормой. Статистическая сумма определяется как след оператора $\exp \left\{2 \pi i \tau L_{0}+\right.$ $\left.2 \pi i \sum \nu_{i} h_{i}\right\}$, где $h_{i}$ - базис подалгебры Картана алгебры симметрии ${ }^{1)}$. Таким образом, для нахождения статистической суммы теории достаточно знать характеры имеюшихся в теории неприводимых представлений алгебры симметрии.

В этой статье обсуждаются конформные теории поля с аффинной $s l(2)$ и $N=2$ суперконформной алгебрами симметрии. Оказывается, что по любой модели с $\widehat{s l}(2)$-симметрией можно однозначно построить модель с $N=2$ симметрией, причем состояния с нулевой нормой в одной теории могут быть получены из состояний с нулевой нормой в другой теории и наоборот (впервые этот факт был отмечен в [12]). Статистические суммы двух теорий тоже могут быть получены одна из другой. Таким образом, теории с $N=2$ и $\widehat{s l}(2)$-алгебрами симметрии находятся во взаимно однозначном соответствии, и результаты, полученные для одной из них, верны и для другой. В основе этого факта лежит эквивалентность категорий цепей представлений алгебры $\widehat{s l}(2)$ и суперконформной алгебры $N=2$, установленная в [13]. В настоящей работе дается конформно-полевое описание результатов [13], а также устанавливаются формулы, связываюшие статистические суммы двух теорий.

В разделе 2 дается описание теорий с $\widehat{s l}(2)$-алгеброй симметрии: примарных состояний, состояний с нулевой нормой и статистических сумм. В разделе 3 определяются $N=2$ суперконформные теории поля и описываются их примарные состояния, статистические суммы и состояния с нулевой нормой. В разделе 4 устанавливается связь между алгебрами симметрии двух теорий. Наконец, в разделе 5 находятся соотношения, связываюшие состояния с нулевой нормой двух теорий, и выводятся формулы, выражающие статистические суммы одной теории через статистические суммы другой.

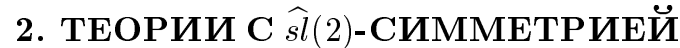

Наличие в теории поля симметрии $\widehat{s l}(2)$ уровня $k$ означает, что среди полей теории имеются токи $J^{ \pm}(z)$ и $J^{0}(z)$ с операторными разложениями

$$
\begin{aligned}
J^{0}(z) J^{ \pm}(w) & = \pm \frac{J^{ \pm}}{z-w}, \quad J^{0}(z) J^{0}(w)=\frac{k / 2}{(z-w)^{2}}, \\
J^{+}(z) J^{-}(w) & =\frac{k}{(z-w)^{2}}+\frac{2 J^{0}}{z-w} .
\end{aligned}
$$

Известно, что такая теория обладает конформной симметрией, определяемой конструкцией Сугавары, и сугаваровский тензор энергии-импульса имеет вид

$$
T^{\text {Sug }}=\frac{1}{k+2}\left(J^{0} J^{0}+\frac{1}{2}\left(J^{+} J^{-}+J^{-} J^{+}\right)\right) .
$$

Здесь и далее предполагается, что $k \neq-2$. Относительно сугаваровского тензора энергии-импульса токи $J^{ \pm}(z)$ и $J^{0}(z)$ имеют конформные размерности 1 , откуда следуют их

\footnotetext{
1) Антиголоморфная часть опущена.
} 
разложения в ряды Лорана

$$
J^{ \pm, 0}(z)=\sum_{n \in \mathbb{Z}} J_{n}^{ \pm, 0} z^{-n-1}
$$

Ряд Лорана по $z$ для сугаваровского тензора энергии-импульса выглядит так:

$$
T^{\mathrm{Sug}}(z)=\sum_{n \in \mathbb{Z}} L_{n}^{\mathrm{Sug}} z^{-n-2} .
$$

Примарные по отношению к $\widehat{s l}(2)$-алгебре состояния $|j, k\rangle_{s l(2)}$ определяются соотношениями

$$
\begin{aligned}
J_{\geq 0}^{+}|j, k\rangle_{s l(2)} & =J_{\geq 1}^{0}|j, k\rangle_{s l(2)}=J_{\geq 1}^{-}|j, k\rangle_{s l(2)}=0, \\
J_{0}^{0}|j, k\rangle_{s l(2)} & =j|j, k\rangle_{s l(2)}, \quad j, k \in \mathbb{C} .
\end{aligned}
$$

Конформную размерность примарного состояния найти легко:

$$
L_{0}^{\mathrm{Sug}}|j, k\rangle_{s l(2)}=\Delta_{j}|j, k\rangle_{s l(2)}=\frac{j(j+1)}{(k+2)}|j, k\rangle_{s l(2)} .
$$

Пространство состояний теории представляет собой прямую сумму секторов суперотбора $\mathcal{I}_{j, k}$, являюшихся неприводимыми представлениями алгебры $\widehat{s l}(2)$; каждое пространство $\mathcal{I}_{j, k}$ получается из примарного состояния $|j, k\rangle_{s l(2)}$ действием операторов рождения из $\widehat{s l}(2)$ (т.е. тех операторов, которые не входят в (2.3)). Отметим еще, что примарные поля действуют между различными секторами суперотбора $\mathcal{I}_{j, k}$.

Статистическая сумма этой теории может быть построена из следов оператора $\exp \left\{2 \pi i \tau L_{0}^{\text {Sug }}-2 \pi i \nu J_{0}^{0}\right\}$ :

$$
\chi_{j, k}^{s l(2)}(\nu, \tau)=\operatorname{Tr}_{\mathcal{I}_{j, k}}\left(\exp \left\{2 \pi i \tau L_{0}^{\text {Sug }}-2 \pi i \nu J_{0}^{0}\right\}\right)
$$

вычисленных в каждом из секторов суперотбора.

Конформная теория с $\widehat{s l}(2)$-алгеброй симметрии допускает преобразование спектрального потока [14]

$$
\mathcal{U}_{\theta}: \quad J_{n}^{+} \mapsto J_{n+\theta}^{+}, \quad J_{n}^{-} \mapsto J_{n-\theta}^{-}, \quad J_{n}^{0} \mapsto J_{n}^{0}+\frac{k}{2} \theta \delta_{n, 0}, \quad \theta \in \mathbb{Z},
$$

которое не меняет операторных разложений (2.1).

Будучи примененным к примарным состояниям $|j, k\rangle_{s l(2)}$, преобразование спектрального потока (2.6) дает твистованные примарные состояния $|j, k ; \theta\rangle_{s l(2)}$, удовлетворяюшие следуюшим условиям:

$$
\begin{aligned}
J_{\geq \theta}^{+}|j, k ; \theta\rangle_{s l(2)} & =J_{\geq 1}^{0}|j, k ; \theta\rangle_{s l(2)}=J_{\geq-\theta+1}^{-}|j, k ; \theta\rangle_{s l(2)}=0, \\
\left(J_{0}^{0}+\frac{k}{2} \theta\right)|j, k ; \theta\rangle_{s l(2)} & =j|j, k ; \theta\rangle_{s l(2)} .
\end{aligned}
$$


Можно также заметить, что преобразование спектрального потока эквивалентно добавлению к тензору энергии-импульса поля $\theta J^{0}(z) / z$. При этом конформные размерности полей $J^{ \pm}(z)$ меняются соответственно на $\pm \theta$.

Вследствие наличия в модели преобразования спектрального потока разумно считать полное пространство состояний теории прямой суммой секторов суперотбора $\mathcal{I}_{j, k ; \theta}$ при всех $\theta \in \mathbb{Z}$, а не только при $\theta=0$. В случае $k \in \mathbb{N}$, который обычно и рассматривается, добавление твистованных примарных состояний не дает ничего нового из-за того, что интегрируемые представления инвариантны относительно преобразований с $\theta \in 2 \mathbb{Z}$, в то время как преобразование спектрального потока с $\theta \in 2 \mathbb{Z}+1$ отображает одно интегрируемое представление в другое. Другое дело, когда $k \in \mathbb{Q}$. В этом случае добавление примарных состояний со всеми $\theta \in \mathbb{Z}$ расширяет теорию. В дальнейшем будут рассматриваться модели, содержашие $\mathcal{I}_{j, k ; \theta}$ при всех $\theta \in \mathbb{Z}$.

Статистическую сумму твистованного сектора суперотбора $\mathcal{I}_{j, k ; \theta}$ можно вычислить, зная статистическую сумму (2.5):

$$
\begin{aligned}
\chi_{j, k ; \theta}^{s l(2)}(\nu, \tau) & =\operatorname{Tr}_{\mathcal{I}_{j, k ; \theta}} \exp \left\{2 \pi i \tau L_{0}^{\mathrm{Sug}}-2 \pi i \nu J_{0}^{0}\right\}= \\
& =e^{2 \pi i\left(\tau \frac{k}{4} \theta^{2}+\nu \frac{k}{2} \theta\right)} \operatorname{Tr}_{\mathcal{I}_{j, k}} \exp \left\{2 \pi i \tau L_{0}^{\mathrm{Sug}}-2 \pi i(\nu+\theta \tau) J_{0}^{0}\right\}= \\
& =e^{2 \pi i\left(\tau \frac{k}{4} \theta^{2}+\nu \frac{k}{2} \theta\right)} \chi_{j, k}^{s l(2)}(\nu+\theta \tau, \tau) .
\end{aligned}
$$

Равенство (2.8) выполняется для характера любого представления алгебры $\widehat{s l}(2)$ из категории $\mathcal{O}[15]$.

Как обсуждалось во введении, уравнения для корреляционных функций теории получаются приравниванием к нулю состояний с нулевой нормой. Такие состояния имеются, если и только если [16] либо $j=\mathbf{j}^{+}(r, s, k)$, либо $j=\mathbf{j}^{-}(r, s, k)$, где

$$
\begin{aligned}
\mathbf{j}^{+}(r, s, k) & =\frac{r-1}{2}-(k+2) \frac{s-1}{2}, \\
\mathbf{j}^{-}(r, s, k) & =-\frac{r+1}{2}+(k+2) \frac{s}{2},
\end{aligned}
$$

Известны также явные формулы для всех таких состояний [17], полученные Маликовым, Фейгином, Фуксом (MFF),

$$
\begin{aligned}
\left|\mathrm{MFF}^{+}(r, s, k)\right\rangle= & \left(J_{0}^{-}\right)^{r+(s-1)(k+2)}\left(J_{-1}^{+}\right)^{r+(s-2)(k+2)}\left(J_{0}^{-}\right)^{r+(s-3)(k+2)} \times \cdots \\
& \cdots \times\left(J_{-1}^{+}\right)^{r-(s-2)(k+2)}\left(J_{0}^{-}\right)^{r-(s-1)(k+2)}\left|\mathbf{j}^{+}(r, s, k), k\right\rangle_{s l(2)}, \\
\left|\mathrm{MFF}^{-}(r, s, k)\right\rangle= & \left(J_{-1}^{+}\right)^{r+(s-1)(k+2)}\left(J_{0}^{-}\right)^{r+(s-2)(k+2)}\left(J_{-1}^{+}\right)^{r+(s-3)(k+2)} \times \cdots \\
& \cdots \times\left(J_{0}^{-}\right)^{r-(s-2)(k+2)}\left(J_{-1}^{+}\right)^{r-(s-1)(k+2)}\left|\mathbf{j}^{-}(r, s, k), k\right\rangle_{s l(2)},
\end{aligned}
$$

где нецелые степени операторов $J_{n}^{ \pm}$понимаются в смысле "аналитического продолжения". Это означает, что с формулами (2.10) следует работать, как с формальными вы-

2 Теоретическая и математическая физика, т. 115, № 1, 1998 г. 
ражениями, про которые известно, что

$$
\begin{aligned}
\left(J_{0}^{-}\right)^{\alpha} J_{m}^{+}= & -\alpha(\alpha-1) J_{m}^{-}\left(J_{0}^{-}\right)^{\alpha-2}-2 \alpha J_{m}^{0}\left(J_{0}^{-}\right)^{\alpha-1}+J_{m}^{+}\left(J_{0}^{-}\right)^{\alpha} \\
\left(J_{0}^{-}\right)^{\alpha} J_{m}^{0}= & \alpha J_{m}^{-}\left(J_{0}^{-}\right)^{\alpha-1}+J_{m}^{0}\left(J_{0}^{-}\right)^{\alpha} \\
\left(J_{-1}^{+}\right)^{\alpha} J_{m}^{-}= & -\alpha(\alpha-1) J_{m-2}^{+}\left(J_{-1}^{+}\right)^{\alpha-2}-k \alpha \delta_{m-1,0}\left(J_{-1}^{+}\right)^{\alpha-1}+ \\
& +2 \alpha J_{m-1}^{0}\left(J_{-1}^{+}\right)^{\alpha-1}+J_{m}^{-}\left(J_{-1}^{+}\right)^{\alpha} \\
\left(J_{-1}^{+}\right)^{\alpha} J_{m}^{0}= & -\alpha J_{m-1}^{+}\left(J_{-1}^{+}\right)^{\alpha-1}+J_{m}^{0}\left(J_{-1}^{+}\right)^{\alpha}
\end{aligned}
$$

Эти формулы выводятся при целых положительных $\alpha$ и затем постулируются для любых комплексных $\alpha$. Подробности см. в [17], где также доказывается, что с помощью (2.11) выражения (2.10) могут быть приведены к полиномиальному по операторам $J_{n}^{ \pm, 0}$ виду.

Очевидно, что состояния с нулевой нормой, соответствующие твистованным примарным состояниям, получаются из стандартных (2.10) действием преобразования спектрального потока.

\section{3. ТЕОРИИ С $N=2$ СУПЕРКОНФОРМНОЙ СИММЕТРИЕЙ}

Перейдем теперь к двумерным теориям, обладаюшим $N=2$ суперконформной симметрией. В таких теориях по определению должны присутствовать четыре тока: тензор энергии-импульса $\mathcal{T}(z)$, два примарных суперполя $\mathcal{Q}(z)$ и $\mathcal{G}(z)$ и $U(1)$-ток $\mathcal{H}(z)$. Эти четыре поля имеют следующие операторные разложения:

$$
\begin{aligned}
\mathcal{T}(z) \mathcal{T}(w) & =\frac{2 \mathcal{T}(w)}{(z-w)^{2}}+\frac{\partial \mathcal{T}}{z-w}, & \mathcal{H}(z) \mathcal{H}(w) & =\frac{c / 3}{(z-w)^{2}}, \\
\mathcal{T}(z) \mathcal{G}(w) & =\frac{2 \mathcal{G}(w)}{(z-w)^{2}}+\frac{\partial \mathcal{G}}{z-w}, & \mathcal{T}(z) \mathcal{Q}(w) & =\frac{\mathcal{Q}(w)}{(z-w)^{2}}+\frac{\partial \mathcal{Q}}{z-w}, \\
\mathcal{T}(z) \mathcal{H}(w) & =\frac{c / 3}{(z-w)^{3}}+\frac{\mathcal{H}(w)}{(z-w)^{2}}+\frac{\partial \mathcal{H}}{z-w}, & \mathcal{H}(z) \mathcal{G}(w) & =\frac{\mathcal{G}}{z-w}, \\
\mathcal{Q}(z) \mathcal{G}(w) & =\frac{c / 3}{(z-w)^{3}}-\frac{\mathcal{H}(w)}{(z-w)^{2}}+\frac{\mathcal{T}}{z-w}, & \mathcal{H}(z) \mathcal{Q}(w) & =-\frac{\mathcal{Q}}{z-w} .
\end{aligned}
$$

Операторные разложения (3.1) записаны для так называемого топологического варианта $N=2$ алгебры. Центральный заряд $c$ удобно параметризовать следующим образом: $c=3(t-2) / t$. Поля $\mathcal{T}(z), \mathcal{H}(z), \mathcal{Q}(z)$ и $\mathcal{G}(z)$ разлагаются в ряды Лорана по переменной $z$ :

$$
\begin{array}{ll}
\mathcal{T}(z)=\sum_{n \in \mathbb{Z}} L_{n} z^{-n-2}, & \mathcal{H}(z)=\sum_{n \in \mathbb{Z}} H_{n} z^{-n-1}, \\
\mathcal{Q}(z)=\sum_{n \in \mathbb{Z}} Q_{n} z^{-n-1}, & \mathcal{G}(z)=\sum_{n \in \mathbb{Z}} G_{n} z^{-n-2} .
\end{array}
$$

Примарные состояния в такой теории определяются условиями

$$
\begin{gathered}
Q_{\geq 1}|h, \ell, t\rangle=G_{\geq 0}|h, \ell, t\rangle=L_{\geq 1}|h, \ell, t\rangle=H_{\geq 1}|h, \ell, t\rangle=0 \\
H_{0}|h, \ell, t\rangle=h|h, \ell, t\rangle, \quad L_{0}|h, \ell, t\rangle=\ell|h, \ell, t\rangle .
\end{gathered}
$$


Будем называть такие состояния массивными примарными состояниями.

В теории с $N=2$ суперконформной симметрией имеется преобразование спектрального потока $[5,18]$

$$
\begin{array}{rlrl}
L_{n} & \mapsto L_{n}+\theta H_{n}+\frac{c}{6}\left(\theta^{2}+\theta\right) \delta_{n, 0}, & H_{n} & \mapsto H_{n}+\frac{c}{3} \theta \delta_{n, 0}, \\
Q_{n} & \mapsto Q_{n-\theta}, & G_{n} \mapsto G_{n+\theta},
\end{array}
$$

не меняющее операторных разложений (3.1). Применяя преобразование (3.5) к примарным состояниям (3.4), получаем твистованные примарные состояния, определяемые условиями

$$
\begin{gathered}
L_{m}|h, \ell, t ; \theta\rangle=0, \quad m \geq 1, \quad Q_{\lambda}|h, \ell, t ; \theta\rangle=0, \quad \lambda \in-\theta+\mathbb{N}, \\
H_{m}|h, \ell, t ; \theta\rangle=0, \quad m \geq 1, \quad G_{\nu}|h, \ell, t ; \theta\rangle=0, \quad \nu=\theta+\mathbb{N}_{0}, \\
\left(H_{0}+\frac{c}{3} \theta\right)|h, \ell, t ; \theta\rangle=h|h, \ell, t ; \theta\rangle, \\
\left(L_{0}+\theta H_{0}+\frac{c}{6}\left(\theta^{2}+\theta\right)\right)|h, \ell, t ; \theta\rangle=\ell|h, \ell, t ; \theta\rangle .
\end{gathered}
$$

Пространство состояний теории - это прямая сумма секторов суперотбора $\mathcal{J}_{h, \ell, t ; \theta}$, каждый из которых несет неприводимое представление $N=2$ суперконформной алгебры, определяемое условиями (3.6).

Статистическая сумма находится аналогично $\widehat{s l}(2)$-случаю:

$$
\begin{aligned}
\chi_{h, \ell, t ; \theta}^{N=2}(\nu, \tau) & =\operatorname{Tr}_{\mathcal{J}_{h, \ell, t ; \theta}} \exp \left\{2 \pi i \tau L_{0}-2 \pi i \nu H_{0}\right\}= \\
& =\exp \left\{2 \pi i\left(\tau \frac{c}{6}\left(\theta^{2}+\theta\right)-\nu \frac{c}{3} \theta\right)\right\} \operatorname{Tr}_{\mathcal{J}_{h, \ell, t}} \exp \left\{2 \pi i \tau L_{0}-2 \pi i(\nu-\theta \tau) H_{0}\right\}= \\
& =\exp \left\{2 \pi i\left(\tau \frac{c}{6}\left(\theta^{2}+\theta\right)-\nu \frac{c}{3} \theta\right)\right\} \chi_{h, \ell, t}^{N=2}(\nu-\theta \tau, \tau)
\end{aligned}
$$

где опять для вычисления следов по твистованным секторам суперотбора достаточно знать их для нетвистованных (т.е. при $\theta=0$ ).

Можно также рассмотреть теорию с более узким множеством примарных состояний, ограничившись так называемыми топологическими примарными состояниями, которые определяются условиями аннигилящии, более жесткими чем (3.6):

$$
\begin{aligned}
& L_{m}|h, t ; \theta\rangle_{\text {top }}=0, \quad m \geq 1, \quad Q_{\lambda}|h, t ; \theta\rangle_{\text {top }}=0, \quad \lambda \in-\theta+\mathbb{N}_{0}, \quad \theta \in \mathbb{Z} . \\
& H_{m}|h, t ; \theta\rangle_{\text {top }}=0, \quad m \geq 1, \quad G_{\nu}|h, t ; \theta\rangle_{\text {top }}=0, \quad \nu \in \theta+\mathbb{N}_{0},
\end{aligned}
$$

Состояние $|h, t ; \theta\rangle_{\text {top }}$ является также собственным для гамильтониана $L_{0}$ и оператора заряда $H_{0}$ :

$$
\begin{aligned}
\left(H_{0}+\frac{c}{3} \theta\right)|h, t ; \theta\rangle_{\mathrm{top}} & =h|h, t ; \theta\rangle_{\mathrm{top}} \\
\left(L_{0}+\theta H_{0}+\frac{c}{6}\left(\theta^{2}+\theta\right)\right)|h, t ; \theta\rangle_{\mathrm{top}} & =0 .
\end{aligned}
$$


Все пространство состояний строится аналогично предыдущему случаю как прямая сумма топологических секторов суперотбора $\mathcal{C}_{h, t ; \theta}$.

Корреляционные функции теории могут быть найдены из уравнений, получающихся приравниванием к нулю состояний с нулевой нормой. Условие наличия таких состояний определяется нулями детерминанта Каца [19]. Рассмотрим сначала теорию, основанную на топологических примарных состояниях (3.8). В такой теории имеются состояния с нулевой нормой, если и только если [13] $h=\mathbf{h}^{+}(r, s, t)$ или $h=\mathbf{h}^{-}(r, s, t)$, где

$$
\mathbf{h}^{+}(r, s, t)=-\frac{r-1}{t}+s-1, \quad \mathbf{h}^{-}(r, s, t)=\frac{r+1}{t}-s, \quad r, s \in \mathbb{N}, \quad t \in \mathbb{C} .
$$

Как и в случае $\widehat{s l}(2)$, для состояний с нулевой нормой можно написать явные формулы, аналогичные (2.10). Для этого необходимо ввести продолженные операторы $q(a, b)$ и $g(a, b)$, имеюшие интуитивный смысл произведений мод суперполей [20]

$$
q(a, b)=\prod_{m=a}^{b} \mathcal{Q}_{m} \quad \text { и } g(a, b)=\prod_{m=a}^{b} \mathcal{G} .
$$

Конечно, это определение имеет смысл, только когда $a$ и $b$ целые и $b-a+1 \geq 0$. Для того чтобы определить такие операторы для любых $a, b \in \mathbb{C}$, нужно продолжить соотношения, которым удовлетворяют $q(a, b)$ и $g(a, b)$, при $a, b \in \mathbb{Z}$ и $b-a+1 \geq 0$, как это сделано в [20]. В качестве примера таких соотношений приведем "принцип Паули"

$$
G_{a} g(b, c)=0, \quad Q_{a} q(b, c)=0, \quad a-b \in \mathbb{N}_{0} \quad \text { и } \quad a-c \notin \mathbb{N} \text { или } b-c-1 \in \mathbb{N} .
$$

Подробное описание свойств этих операторов можно найти в [20]. В терминах операторов $q(a, b)$ и $g(a, b)$ для всех состояний с нулевой нормой имеются явные формулы [20]

$$
\begin{aligned}
|E(r, s, t)\rangle^{+}= & g(-r,(s-1) t-1) q(-(s-1) t, r-1-t) \ldots g((s-2) t-r, t-1) \times \\
& \times q(-t, r-1-t(s-1)) g((s-1) t-r,-1)\left|\mathbf{h}^{+}(r, s, t), t\right\rangle_{\mathrm{top}}, \\
|E(r, s, t)\rangle^{-}= & q(-r,(s-1) t-1) g(-(s-1) t, r-t-1) \ldots q((s-2) t-r, t-1) \times \\
& \times g(-t, r-1-(s-1) t) q((s-1) t-r,-1)\left|\mathbf{h}^{-}(r, s, t), t\right\rangle_{\mathrm{top}},
\end{aligned}
$$

где $r, s \in \mathbb{N}$ и сомножители в первой строке каждой формулы равны

$$
g(-r-t-m t+s t,-1+m t) q(-m t, r-1+m t-s t), \quad s-1 \geq m \geq 1,
$$

и

$$
q(-r-t-m t+s t,-1+m t) g(-m t, r-1+m t-s t), \quad s-1 \geq m \geq 1 .
$$

Как показано в [21], с помощью свойств операторов $q$ и $g$ выражения (3.12) и (3.13) могут быть приведены к полиномиальной по модам токов (3.2) форме. 
В случае более широкой теории, содержашей массивные примарные состояния, векторы с нулевой нормой сушествуют, если и только если [19] $\ell=\mathbf{1}_{\mathrm{ch}}(n, h, t)$ или $\ell=$ $\mathbf{l}(r, s, h, t)$, где

$$
\mathbf{l}_{\mathrm{ch}}(n, h, t)=n\left(h+\frac{n-1}{t}\right), \quad n \in \mathbb{Z}, \quad t \in \mathbb{C}, \quad h \in \mathbb{C},
$$

и

$$
\mathbf{l}(r, s, h, t)=-\frac{t}{4}\left(h-\mathbf{h}^{-}(r, s, t)\right)\left(h-\mathbf{h}^{+}(r, s+1, t)\right), \quad r, s \in \mathbb{N}, \quad t \in \mathbb{C}, \quad h \in \mathbb{C} .
$$

Для состояний с нулевой нормой снова имеются явные формулы [21]. Когда выполнено условие $\ell=\mathrm{l}_{\mathrm{ch}}(n, h, t)$, такие состояния имеют чрезвычайно простой вид:

$$
|E(n, h, t)\rangle_{\mathrm{ch}}= \begin{cases}Q_{n} \ldots Q_{0}\left|h, \mathbf{l}_{\mathrm{ch}}(n, h, t), t\right\rangle, & n \leq 0, \\ G_{-n} \ldots G_{-1}\left|h, \mathbf{l}_{\mathrm{ch}}(n, h, t), t\right\rangle, & n \geq 1 .\end{cases}
$$

В случае же $\ell=\mathbf{l}(r, s, h, t)$ снова требуются продолженные операторы $q(a, b)$ и $g(a, b)$, с помощью которых состояния с нулевой нормой записываются в виде

$$
\begin{aligned}
|S(r, s, h, t)\rangle^{-}= & g\left(-r s, r+\theta^{-}(r, s, h, t)-1\right) \mathcal{E}^{-, \theta^{-}(r, s, h, t)}(r, s, t) \times \\
& \times g\left(\theta^{-}(r, s, h, t),-1\right)|h, \mathbf{l}(r, s, h, t), t\rangle, \\
|S(r, s, h, t)\rangle^{+}= & q\left(1-r s, r-\theta^{+}(r, s, h, t)-1\right) \mathcal{E}^{+, \theta^{+}(r, s, h, t)}(r, s, t) \times \\
& \times q\left(-\theta^{+}(r, s, h, t), 0\right)|h, \mathbf{l}(r, s, h, t), t\rangle,
\end{aligned}
$$

где $\mathcal{E}^{ \pm, \theta}(r, s, t)$ - операторы, которые порождают из примарных состояний состояния с нулевой нормой (3.12) и (3.13), подвергнутые преобразованию спектрального потока с параметром $\theta$, равным

$$
\theta^{-}(r, s, h, t)=\frac{t}{2}\left(h-\mathbf{h}^{-}(r, s, t)\right), \quad \theta^{+}(r, s, h, t)=\frac{t}{2}\left(h-1-\mathbf{h}^{+}(r, s, t)\right) .
$$

Обычно оказывается, что (3.18) можно получить из (3.19) действием операторов $Q_{n}$ и, наоборот, (3.19) может быть получено из (3.18) действием операторов $G_{n}$, т.е. уравнения на корреляционные функции, полученные из (3.18) и (3.19), обычно оказьваются эквивалентными. Вопросы теории представлений, связанные с наличием нескольких состояний с нулевой нормой и эквивалентностью уравнений, следующих из (3.18) и (3.19), подробно описаны в [22].

\section{4. ОПЕРАТОРНОЕ ОТОБРАЖЕНИЕ МЕЖДУ ТЕОРИЯМИ}

Обратимся теперь к связи моделей, описанных в двух предыдущих разделах. Она дается операторной конструкцией $\left.{ }^{2}\right)[\widehat{s l}(2)] \otimes[B C]$, где $[B C]-$ это пара духов спина -1 , называемых также $B C$-системой, с операторным разложением вида

\footnotetext{
${ }^{2)}$ Которую можно рассматривать как конструкцию Казамы-Сузуки, связанную с простейшим косетом $\widehat{s l}(2)_{k} \oplus u(1) / u(1)$.
} 


$$
B(z) C(w)=\frac{1}{z-w}
$$

и тензором энергии-импульса $T^{\mathrm{gh}}=-B \partial C$ (где $T^{\mathrm{gh}}-$ духовый тензор энергии-импульса). Будем использовать разложения по модам

$$
B(z)=\sum_{n \in \mathbb{Z}} B_{n} z^{-n-1} \quad \text { и } \quad C(z)=\sum_{n \in \mathbb{Z}} C_{n} z^{-n} .
$$

Обозначим через $\Omega$ фоковское пространство, порожденное $B C$-системой из вакуума $|0\rangle_{\mathrm{gh}}$, определенного условиями аннигиляции

$$
C_{\geq 1}|0\rangle_{\mathrm{gh}}=B_{\geq 0}|0\rangle_{\mathrm{gh}}=0 .
$$

Определенный таким образом вакуум есть проективно-инвариантное состояние [9]. В пространстве Фока $\Omega$, однако, есть много других эквивалентных вакуумов $|\lambda\rangle_{\mathrm{gh}}$, которые удовлетворяют условиям

$$
C_{\geq 1-\lambda}|\lambda\rangle_{\mathrm{gh}}=B_{\geq \lambda}|\lambda\rangle_{\mathrm{gh}}=0,
$$

откуда следует, что

$$
\begin{aligned}
(B C)_{0}|\lambda\rangle_{\mathrm{gh}} & =-\lambda|\lambda\rangle_{\mathrm{gh}}, \\
L_{0}^{\mathrm{gh}}|\lambda\rangle_{\mathrm{gh}} & =\frac{1}{2} \lambda(\lambda-1)|\lambda\rangle_{\mathrm{gh}} .
\end{aligned}
$$

Статистическая сумма $B C$-системы имеет вид

$$
\begin{aligned}
\chi^{\mathrm{gh}}(\nu, \tau) & =\operatorname{Tr}_{\Omega} \exp \left\{2 \pi i \tau L_{0}^{\mathrm{gh}}+2 \pi i \nu(B C)_{0}\right\}= \\
& =\prod_{m=0}^{\infty}\left(1+e^{2 \pi i m \tau-2 \pi i \nu}\right) \prod_{m=1}^{\infty}\left(1+e^{2 \pi i m \tau+2 \pi i \nu}\right)= \\
& =e^{\frac{\pi i \tau}{6}} e^{\pi i \nu} \frac{\vartheta_{2}(\nu, \tau)}{\eta(\tau)},
\end{aligned}
$$

где $\vartheta_{2}(\nu, \tau)$ и $\eta(\tau)$ - соответственно тэта-функция и функция Дедекинда, определяемые рядами

$$
\vartheta_{2}(\nu \mid \tau)=\sum_{m=-\infty}^{\infty} e^{\pi i\left(m-\frac{1}{2}\right)^{2} \tau} e^{\pi i(2 m-1) \nu}, \quad \eta(\tau)=e^{\frac{\pi i \tau}{12}} \prod_{m=1}^{\infty}\left(1-e^{2 \pi i m \tau}\right) .
$$

Определенная выше $B C$-система дает возможность стандартным образом $[23,24]$ построить генераторы $N=2$ алгебры. Нечетные генераторы $Q$ и $G$ даются выражениями

$$
Q=C J^{+}, \quad G=\frac{2}{k+2} B J^{-},
$$


тогда как $U(1)$-ток и тензор энергии-импульса $N=2$ теории принимают вид

$$
\begin{gathered}
H=\frac{k}{k+2} B C-\frac{2}{k+2} J^{0} \\
T=\frac{1}{k+2}\left(J^{+} J^{-}\right)-\frac{k}{k+2} B \partial C-\frac{2}{k+2} B C J^{0} .
\end{gathered}
$$

Сингулярные части в операторных разложениях генераторов (4.7), (4.8) и (4.9) удовлетворяют операторным разложениям (3.1). Центральный заряд $N=2$ теории выражается через уровень алгебры $\widehat{s l}(2)$ следуюшим образом:

$$
c=\frac{3 k}{k+2}
$$

В системе $\widehat{s l}(2) \otimes[B C]$ присутствует еше свободное скалярное поле

$$
I^{+}=\sqrt{\frac{2}{k+2}}\left(B C+J^{0}\right),
$$

не имеющее особенностей в операторных разложениях со всеми $N=2$ токами (4.7)-(4.9). Разлагая это поле по модам

$$
I^{+}(z)=\sum_{n \in \mathbb{Z}} I_{n}^{+} z^{-n-1}
$$

мы получаем алгебру Гейзенберга $\left[I_{m}^{+}, I_{n}^{+}\right]=m \delta_{m+n, 0}$. Тензор энергии-импульса поля $I^{+}$имеет вид

$$
T^{+}=\frac{1}{2}\left(I^{+}\right)^{2}-\frac{1}{\sqrt{2(k+2)}} \partial I^{+} .
$$

Фоковское пространство $\mathcal{H}_{p}^{+}$поля $I^{+}$порождается из вакуума, удовлетворяющего соотношениям

$$
I_{\geq 1}|p\rangle^{+}=0, \quad I_{0}|p\rangle^{+}=p|p\rangle^{+} .
$$

Статистическая сумма теории свободного скалярного поля $I^{+}$есть

$$
\begin{aligned}
\chi_{p, k}^{+}(\nu, \tau) & =\operatorname{Tr}_{\mathcal{H}_{p}^{+}} \exp \left\{2 \pi i \tau L_{0}^{+}-2 \pi i \nu I_{0}^{+}\right\}= \\
& =\exp \left\{-2 \pi i \nu p+\pi i \tau p\left(p+\sqrt{\frac{2}{k+2}}\right)+\frac{\pi i \tau}{12}\right\} \frac{1}{\eta(\tau)} .
\end{aligned}
$$

Равенство числа степеней свободы в системах $[\widehat{s l}(2)] \otimes[B C]$ и $[N=2] \oplus\left[I^{+}\right]$выражается в виде соотношений полноты для тензоров энергии-импульса и $U(1)$-токов

$$
T_{\mathrm{Sug}}+T_{\mathrm{gh}}=T+T^{+}
$$

(где $T$ - тензор энергии-импульса (4.9)) и

$$
J^{0}-B C=-2 H+\frac{k-2}{\sqrt{2(k+2)}} I^{+} .
$$


Исходя из $N=2$ теории тоже можно построить операторную конструкцию, со-

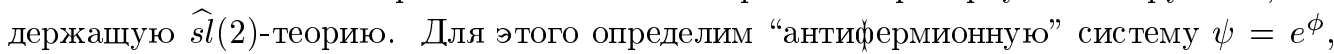
$\psi^{*}=e^{-\phi}$, где $\phi-$ свободное скалярное поле с сигнатурой -1 :

$$
\phi(z) \phi(w)=-\ln (z-w)
$$

При этом тензор энергии-импульса имеет вид

$$
T_{\phi}=-\frac{1}{2} \partial \phi \partial \phi+\frac{1}{2} \partial^{2} \phi
$$

По отношению к этому тензору энергии-импульса антифермионы $\psi$ и $\psi^{*}$ имеют конформные размерности соответственно 0 и -1 , что фиксирует их разложения по модам

$$
\psi(z)=\sum_{n=-\infty}^{\infty} \frac{\psi_{n}}{z^{n}}, \quad \psi^{*}(z)=\sum_{n=-\infty}^{\infty} \frac{\psi_{n}^{*}}{z^{n-1}}, \quad \partial \phi(z)=\sum_{n=-\infty}^{\infty} \frac{\phi_{n}}{z^{n+1}} .
$$

Операторы $\psi$ и $\psi^{*}$ естественно действуют на прямой сумме

$$
\Xi=\bigoplus_{n \in \mathbb{Z}} \mathcal{H}_{n}
$$

фоковских пространств $\mathcal{H}_{n}$ с вакуумами $|n\rangle_{\phi}$, удовлетворяющими условиям аннигиляции

$$
\begin{aligned}
\phi_{m}|n\rangle_{\phi} & =0, \quad m \geq 1, \\
\psi_{m}|n\rangle_{\phi} & =0, \quad m \geq n+1, \\
\psi_{m}^{*}|n\rangle_{\phi} & =0, \quad m \geq-n+2, \\
\phi_{0}|n\rangle_{\phi} & =-n|n\rangle_{\phi} .
\end{aligned}
$$

Из $N=2$ токов и антифермионной системы можно сконструировать $s l(2)$-токи:

$$
\begin{aligned}
J^{+} & =Q \psi, \quad J^{-}=\frac{3}{3-c} G \psi^{*}, \\
J^{0} & =-\frac{3}{3-c} H+\frac{c}{3-c} \partial \phi, \quad c \neq 3 .
\end{aligned}
$$

Сингулярные части в операторных разложениях токов (4.22) совпадают с (2.1), причем уровень $\widehat{s l}(2)$ равен $k=2 c /(3-c)$.

Сугаваровский тензор энергии-импульса (2.2) вычисляется как

$$
T^{\mathrm{Sug}}=T+\frac{1}{2} \partial H+\frac{k+2}{4} H H-\frac{3}{3-c} H \partial \phi+\frac{k}{4} \partial \phi \partial \phi .
$$

Имеется также свободное скалярное поле с сигнатурой -1 , не дающее сингулярностей в операторных разложениях с токами (4.22):

$$
I^{-}=\sqrt{\frac{k+2}{2}}(H-\partial \phi) \text {. }
$$


Моды $I_{n}^{-}$, определенные посредством разложения

$$
I^{-}(z)=\sum_{n=-\infty}^{\infty} I_{n}^{-} z^{-n-1}
$$

образуют алгебру Гейзенберга $\left[I_{n}^{-}, I_{m}^{-}\right]=-n \delta_{m+n, 0}$. Поле $I^{-}$имеет следуюший тензор энергии-импульса:

$$
T^{-}=-\frac{1}{2}\left(I^{-}\right)^{2}-\frac{1}{\sqrt{2(k+2)}} \partial I^{-} .
$$

Фоковское пространство $\mathcal{H}_{q}^{-}$поля $I^{-}$строится из вакуума, удовлетворяюшего условиям

$$
I_{n}^{-}|q\rangle^{-}=0, \quad n \geq 1, \quad I_{0}^{-}|q\rangle^{-}=q|q\rangle^{-}
$$

Наконец, выполняются соотношения полноты для тензоров энергии-импульса и $U(1)$-токов:

$$
T+T_{\phi}=T^{\mathrm{Sug}}+T^{-}
$$

(где $T$ - тензор энергии-импульса $N=2$ теории, $T_{\phi}$ - тензор энергии-импульса поля $\left.I^{-}(4.28)\right)$ и

$$
-2 H+\partial \phi=J^{0}+\left(\frac{k}{2}-1\right) \sqrt{\frac{k+2}{2}} I^{-} .
$$

\section{5. ОТОБРАЖЕНИЕ МЕЖДУ ПРОСТРАНСТВАМИ СОСТОЯНИЙ}

В предыдущем разделе мы установили соотношение между двумя теориями на уровне алгебр симметрии. Для завершения анализа необходимо установить, как соотносятся пространства состояний и уравнения на корреляционные функции. Поскольку в каждой из теорий пространство состояний представляет собой прямую сумму секторов суперотбора, достаточно выяснить поведение последних.

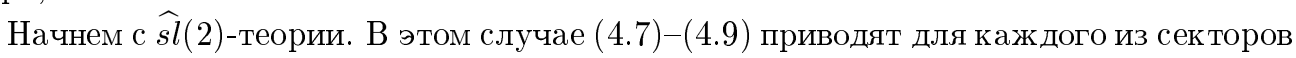
суперотбора $\mathcal{I}_{j, k ; \theta} \mathrm{K}$ равенству

$$
\mathcal{I}_{j, k ; \theta} \otimes \Omega \approx \bigoplus_{m \in \mathbb{Z}} \mathcal{C}_{\frac{-2 j}{k+2}, k+2 ; m-\theta} \otimes \mathcal{H}_{\sqrt{\frac{2}{k+2}}\left(j-\frac{k}{2} \theta-m\right)}
$$

которое связывает их с топологическими секторами суперотбора $\mathcal{C}_{\frac{-2 j}{k+2}, k+2 ; m-\theta}$ в модели с $N=2$ суперконформной симметрией. В то же время формулы (4.22) приводят к равенству

$$
\mathcal{C}_{h, t ; \theta} \otimes \Xi \approx \bigoplus_{n \in \mathbb{Z}} \mathcal{I}_{-\frac{t}{2} h, t-2 ; n-\theta} \otimes \mathcal{H}_{\sqrt{\frac{t}{2}}\left(h-\frac{t-2}{t} \theta+n\right)}^{-}
$$


Доказательства и подробности можно найти в [13]. Состояния с нулевой нормой (2.10), (3.12) и (3.13) в двух теориях соотносятся следуюшим образом:

$$
\begin{gathered}
\left|E\left(r, s, t ; \theta^{\prime}\right)\right\rangle^{+} \otimes\left|\sqrt{\frac{2}{t}}\left(\frac{r-2 \theta^{\prime}-1}{2}-t \frac{s+\theta-1}{2}\right)\right\rangle^{+}= \\
=\left|\mathrm{MFF}^{+}(r, s, t-2 ; \theta)\right\rangle \otimes\left|\theta+\theta^{\prime}-r\right\rangle_{\mathrm{gh}}, \\
\left|E\left(r, s, t ; \theta^{\prime}\right)\right\rangle^{-} \otimes\left|\sqrt{\frac{2}{t}}\left(-\frac{r+2 \theta^{\prime}+1}{2}+t \frac{s-\theta}{2}\right)\right\rangle^{+}= \\
=\left|\mathrm{MFF}^{-}(r, s, t-2 ; \theta)\right\rangle \otimes\left|\theta+\theta^{\prime}+r\right\rangle_{\mathrm{gh}},
\end{gathered}
$$

и в обратную сторону:

$$
\begin{gathered}
\left|\operatorname{MFF}^{+}\left(r, s, t-2 ; \theta^{\prime}\right)\right\rangle \otimes\left|\sqrt{\frac{t}{2}}\left(-\frac{r+2 \theta-1}{2}+s+\theta^{\prime}-1\right)\right\rangle^{-}= \\
=|E(r, s, t ; \theta)\rangle^{+} \otimes\left|\theta+\theta^{\prime}-r\right\rangle_{\phi}, \\
\left|\operatorname{MFF}^{-}\left(r, s, t-2 ; \theta^{\prime}\right)\right\rangle \otimes\left|\sqrt{\frac{t}{2}}\left(\frac{r-2 \theta+1}{2}-s+\theta^{\prime}\right)\right\rangle^{-}= \\
=|E(r, s, t ; \theta)\rangle^{-} \otimes\left|\theta+\theta^{\prime}+r\right\rangle_{\phi} .
\end{gathered}
$$

Итак, зная корреляционные функции одной теории, можно в принципе вычислить корреляционные функции другой.

Подобную связь можно получить также и для статистических сумм двух теорий. Действительно, из соотношений полноты (4.16) и (4.28), а также формул (5.1) и (5.2) находим

$$
\chi_{j, t ; \theta}^{s l(2)}(\nu, \tau) \chi^{\mathrm{gh}}(\nu, \tau)=\sum_{\theta^{\prime}=-\infty}^{+\infty} \chi_{\frac{-2 j}{t}, t ; \theta^{\prime}}^{N=2}(-2 \nu, \tau) \chi_{\sqrt{\frac{2}{t}}\left(j-\frac{t}{2} \theta-\theta^{\prime}\right), t}^{+}\left(\sqrt{\frac{t}{2}}(1-4 t) \nu, \tau\right),
$$

откуда

$$
\begin{aligned}
& \chi_{j, t ; \theta}^{s l(2)}(\nu, \tau)=\frac{\exp \left\{\pi i\left(\left(\frac{1}{4}+\frac{2 j}{t}+\frac{2 j^{2}}{t}-\theta-2 j \theta+\theta^{2}\right) \tau+\left(1-2 j+\frac{8 j}{t}-2 \theta\right) \nu\right)\right\}}{\vartheta_{2}(\nu, \tau)} \times \\
& \times \sum_{\theta^{\prime}=-\infty}^{+\infty} \exp \left\{\pi i\left(\left(-\frac{2 \theta^{\prime}}{t}-\frac{4 j \theta^{\prime}}{t}+2 \theta \theta^{\prime}+\frac{2 \theta^{\prime 2}}{t}\right) \tau+\left(2 \theta^{\prime}-\frac{8 \theta^{\prime}}{t}\right) \nu\right)\right\} \chi_{\frac{-2 j}{t}, t ; \theta^{\prime}}^{N=2}(-2 \nu, \tau) .
\end{aligned}
$$

Можно также написать аналогичные формулы, выражающие статистические суммы $N=2$ теории через статистические суммы $\widehat{s l}(2)$-теории.

Таким образом, по любой двумерной конформной теории, обладаюшей $\widehat{s l}(2)$-симметрией такой, как описано в разделе 2 , можно построить некоторую двумерную $N=2$ суперконформную теорию, набор примарных состояний которой ограничивается твистованными топологическими состояниями. Обратно, любая двумерная $N=2$ суперконформная теория, все примарные состояния которой являются твистованными топологическими, определяется некоторой теорией с $\widehat{s l}(2)$-симметрией. Итак, эти классы моделей находятся во взаимно однозначном соответствии. 
Однако встает вопрос, какие модели с $\widehat{s l}(2)$-симметрией соответствуют теориям с $N=2$ суперконформной симметрией, содержашим, кроме топологических, еше и массивные примарные состояния (3.6). Оказывается, аналогом массивных примарных состояний в $\widehat{s l}(2)$-теориях являются примарные состояния с более слабыми, чем (2.7), условиями аннигиляции. Эти состояния называются расслабленными ${ }^{3)}$ примарными состояниями и определяются соотношениями

$$
J_{\geq \theta+1}^{+}|j, \Lambda, k ; \theta\rangle_{s l(2)}=J_{\geq 1}^{0}|j, \Lambda, k ; \theta\rangle_{s l(2)}=J_{\geq-\theta+1}^{-}|j, \Lambda, k ; \theta\rangle_{s l(2)}=0,
$$

причем на действие операторов $J_{\theta}^{+}$и $J_{-\theta}^{-}$накладывается связь

$$
J_{-\theta}^{-} J_{\theta}^{+}|j, \Lambda, k ; \theta\rangle_{s l(2)}=\Lambda|j, \Lambda, k ; \theta\rangle_{s l(2)} .
$$

Расслабленное примарное состояние $|j, \Lambda, k ; \theta\rangle_{s l(2)}$ является также собственным для оператора $J_{0}^{0}$ :

$$
\left(J_{0}^{0}+\frac{k}{2} \theta\right)|j, \Lambda, k ; \theta\rangle_{s l(2)}=j|j, \Lambda, k ; \theta\rangle_{s l(2)} .
$$

Пространство состояний такой модели есть прямая сумма секторов суперотбора $\mathcal{D}_{j, \Lambda, k ; \theta}$, каждый из которых порождается из расслабленного примарного состояния $|j, \Lambda, k ; \theta\rangle_{s l(2)}$ действием операторов рождения $J_{\leq \theta-1}^{+}, J_{\leq-\theta-1}^{-}$и $J_{\leq-1}^{0}$.

Каждый из секторов суперотбора $\mathcal{D}_{j, \Lambda, k ; \theta}$ несет неприводимое представление алгебры $\widehat{s l}(2)$, расслабленные примарные поля действуют между различными $\mathcal{D}_{j, \Lambda, k ; \theta}$. Статистическая сумма таких моделей определяется аналогично (2.5) и (2.8) с очевидной заменой $\mathcal{I}_{j, k ; \theta}$ на $\mathcal{D}_{j, \Lambda, k ; \theta}$. Условия появления и явные формулы для состояний с нулевой нормой можно найти в [13]. Формулы (5.1)-(5.6) переписываются с очевидными изменениями в случае моделей, допускающих среди примарных состояний расслабленные/массивные состояния; например, (5.1) принимает вид

$$
\mathcal{D}_{j, \Lambda, k ; \theta} \otimes \Omega \approx \bigoplus_{\lambda \in \mathbb{Z}} \mathcal{J}_{\frac{-2 j}{k+2}, \frac{\Lambda}{k+2}, k+2 ; \lambda-\theta} \otimes \mathcal{H}^{+} \sqrt{\frac{2}{k+2}}\left(j-\frac{k}{2} \theta-\lambda\right)
$$

Таким образом, теории, обладаюшие $\widehat{s l}(2)$ и $N=2$ суперконформной симметриями, находятся во взаимно однозначном соответствии.

\section{6. ОБСУЖДЕНИЕ И ПЕРСПЕКТИВЫ}

В работе показано, что модели двумерной конформной теории поля, обладающие афффинной $s l(2)$ и $N=2$ суперконформной алгебрами симметрии, находятся во взаимно однозначном соответствии. Все результаты, полученные для $\widehat{s l}(2)$-моделей, имеют аналоги в $N=2$ моделях и наоборот. В связи с этим хочется отметить следующие три момента.

Во-первых, из соотношений типа (5.6) между статистическими суммами двух теорий следуют тождества между бесконечными суммами и бесконечными произведениями, в

\footnotetext{
3) По причине более слабых условий аннигиляции.
} 
частности, из соотношения (5.6) и аналогичного обратного соотношения следуют новые соотношения для тэта-функций.

Во-вторых, интересно применить полученное соответствие к теории $N=2$ струн. Оно должно давать связь между БРСТ-когомологиями (физическими состояниями) $N=2$ струны и БРСТ-когомологиями $s l(2)$-модели Весса-Зумино-Виттена.

В-третьих, описанное соответствие, возможно, позволит лучше понять правила слияния обеих теорий. В частности, правила слияния, известные для унитарных теорий с $\widehat{s l}(2)$ и $N=2$ симметриями, могут быть получены друг из друга. Как известно, унитарные представления $\widehat{s l}(2)$-инвариантны при действии преобразования спектрального потока (2.6) с четным параметром $\theta$. Для $N=2$ наблюдается периодичность в теориях типа Ландау-Гинзбурга, а именно, унитарные $N=2$ представления не меняются при преобразовании спектрального потока (3.5) с параметром $\theta=k+2$, где $c=3 k /(k+2)$ и $k \in \mathbb{N}$. Прямой подстановкой можно убедиться, что каждая из формул (5.1) и (5.2) переносит свойство периодичности под действием замены $\theta \mapsto \theta+2$ для $\widehat{s l}(2)$-теорий на периодичность под действием замены $\theta \mapsto \theta+k+2$ для $N=2$ теорий. Таким образом, в унитарных теориях с $\widehat{s l}(2)$ и $N=2$ симметриями правила слияния совпадают с точностью до преобразования спектрального потока. Полезным с точки зрения нахождения полных правил слияния является также введение для $\widehat{s l}(2)$-алгебры представлений расслабленного типа - двойников массивных. Правила слияния в $\widehat{s l}(2)-$ теориях, основанных на представлениях расслабленного типа, должны управляться квантовой универсальной обертывающей алгебры $s \ell(2 \mid 1)$ так же, как правила слияния в моделях, описанных в разделе 2, управляются квантовой универсальной обертывающей алгебры $o s p(1 \mid 2)$. Квантовая универсальная обертывающая алгебра $s \ell(2 \mid 1)_{q}$ лучше подходит для описания правил слияния ${ }^{4)}$ по той причине, что в трехбозонной реализации алгебр $\widehat{s l}(2)$ и $N=2$ алгебра экранируюших операторов в точности образует верхнюю треугольную подалгебру алгебры $s \ell(2 \mid 1)_{q}$.

Автор благодарен А.М. Семихатову за ценные обсуждения и прочтение рукописи, а также И.В. Тютину за замечания по поводу физической интерпретации полученных результатов.

Данная работа была осуществлена при финансовой поддержке РФФИ (проект 96-01-00725).

\section{Список литературы}

[1] E. Witten. Commun. Math. Phys. 1984. V. 92. P. 455.

[2] A. M. Polyakov. Mod. Phys. Lett. A. 1987. V. 2. P. 893.

[3] V. G. Knizhnik, A. M. Polyakov, A. B. Zamolodchikov. Mod. Phys. Lett. A. 1988. V. 3. P. 819.

[4] M. Ademollo et al. Phys. Lett. B. 1976. V. 62. P. 105; Nucl. Phys. B. 1976. V. 111. P. 77; Phys. Lett. B. 1995. V. 363. P. 166.

[5] W. Lerche, C. Vafa, N. P. Warner. Nucl. Phys. B. 1989. V. 324. P. 427.

[6] B. Gato-Rivera, A. M. Semikhatov. Phys. Lett. B. 1992. V. 293. P. 72.

[7] M. Bershadsky, W. Lerche, D. Nemeschansky, N. P. Warner. Nucl. Phys. B. 1993. V. 401. P. 304-347.

\footnotetext{
4) Частное сообщение Б.Л. Фейгина.
} 
[8] A. A. Belavin, A. M. Polyakov, A. B. Zamolodchikov. Nucl. Phys. B. 1984. V. 241. P. 333.

[9] D. H. Friedan, E. J. Martinec, S. H. Shenker. Nucl. Phys. B. 1986. V. 271. P. 93.

[10] V. G. Kač. Covariant form for infinite-dimensional Lie algebras and superalgebras. In: Lect. Notes in Phys. V. 94, 1979. P. 441.

[11] Б. Л. Фейгин, Д.Б. Фукс. Функц. анализ и его прилож. 1982. Т. 16. С. 114.

[12] A. M. Semikhatov. Mod. Phys. Lett. A. 1994. V. 9. P. 1867.

[13] B. L. Feigin, A.M. Semikhatov, I. Yu. Tipunin. Equivalence between Chain Categories of Representations of Affine $s l(2)$ and $N=2$ Superconformal Algebras. hep-th/9701043.

[14] K. Bardakçi, M. B. Halpern. Phys. Rev. D. 1971. V. 3. P. 2493.

[15] V. G. Kač. Infinite dimensional Lie algebras. Cambridge: Cambridge Univ. Press, 1990.

[16] V. G. Kač, D. A. Kazhdan. Adv. Math. 1979. V. 34. P. 97.

[17] Ф. Г. Маликов, Б. Л. Фейгин, Д. Б. Фукс. Функц. анализ и его прилож. 1986. Т. 20. № 2. C. 25 .

[18] A. Schwimmer, N. Seiberg. Phys. Lett. B. 1987. V. 184. P. 191.

[19] W. Boucher, D. Friedan, A. Kent. Phys. Lett. B. 1986. V. 172. P. 316.

[20] A. M. Semikhatov, I. Yu. Tipunin. Int. J. Mod. Phys. A. 1996. V. 11. P. 4597.

[21] A. M. Semikhatov, I. Yu. Tipunin. Singular vectors of the $N=2$ Superconformal Algebra via the Algebraic Continuation Approach. hep-th/9604176.

[22] A. M. Semikhatov, I. Yu. Tipunin. Structure of Verma Modules over the $N=2$ Superconformal Algebra. hep-th/9704111; Commun. Math. Phys. 1998 (to appear).

[23] P. Di Vecchia, J. L. Petersen, M. Yu, H. B. Zheng. Phys. Lett. B. 1986. V. 174. P. 280.

[24] Y. Kazama, H. Suzuki. Nucl. Phys. B. 1989. V. 321. P. 232. 